\title{
On the rate of convergence in the central limit theorem for martingale difference sequences
}

\author{
Lahcen Ouchti \\ LMRS, UMR 6085, université de Rouen, site Colbert, 76821 Mont-Saint-Aignan cedex, France \\ Received 7 July 2003; received in revised form 5 February 2004; accepted 17 March 2004 \\ Available online 11 September 2004
}

\begin{abstract}
We established the rate of convergence in the central limit theorem for stopped sums of a class of martingale difference sequences.

(c) 2004 Elsevier SAS. All rights reserved.
\end{abstract}

\section{Résumé}

On établit la vitesse de convergence dans le théorème limite central pour les sommes arrêtées issues d'une classe de suites de différences de martingale.

(c) 2004 Elsevier SAS. All rights reserved.

MSC: 60G42; 60F05

Keywords: Central limit theorem; Martingale difference sequence; Rate of convergence

\section{Introduction}

Let $\left(X_{i}\right)_{i \in \mathbb{N}}$ be a sequence of random variables defined on a probability space $(\Omega, \mathcal{F}, \mathbb{P})$. We shall say that $\left(X_{k}\right)_{k \in \mathbb{N}}$ is a martingale difference sequence if, for any $k \geqslant 0$

1. $\mathbb{E}\left\{\left|X_{k}\right|\right\}<+\infty$.

2. $\mathbb{E}\left\{X_{k+1} \mid \mathcal{F}_{k}\right\}=0$, where $\mathcal{F}_{k}$ is the $\sigma$-algebra generated by $X_{i}, i \leqslant k$.

For each integer $n \geqslant 1$ and $x$ real number, we denote

E-mail address: lahcen.ouchti@univ-rouen.fr (L. Ouchti). 


$$
\begin{aligned}
& S_{0}=0, \quad S_{n}=\sum_{i=1}^{n} X_{i}, \quad \phi(x)=\frac{1}{\sqrt{2 \pi}} \int_{-\infty}^{x} \exp \left(-\frac{t^{2}}{2}\right) \mathrm{d} t, \quad \sigma_{n-1}^{2}=\mathbb{E}\left\{X_{n}^{2} \mid \mathcal{F}_{n-1}\right\}, \\
& v(n)=\inf \left\{k \in \mathbb{N}^{*}: \sum_{i=0}^{k} \sigma_{i}^{2} \geqslant n\right\}, \quad S_{v(n)}^{2}=\sum_{k=1}^{+\infty} S_{k}^{2} I_{v(n)=k}, \quad \sigma_{v(n)}^{2}=\sum_{k=1}^{+\infty} \sigma_{k}^{2} I_{v(n)=k}, \\
& F_{n}(x)=\mathbb{P}\left(S_{v(n)} \leqslant x \sqrt{n}\right), \quad S_{v(n)}^{\prime}=S_{v(n)}+\sqrt{\gamma(n)} X_{v(n)+1}, \quad H_{n}(x)=\mathbb{P}\left(S_{v(n)}^{\prime} \leqslant x \sqrt{n}\right),
\end{aligned}
$$

and $\gamma(n)$ is a random variable such that

$$
\sum_{i=0}^{\nu(n)-1} \sigma_{i}^{2}+\gamma(n) \sigma_{v(n)}^{2}=n \quad \text { a.s. }
$$

If the random variables $X_{i}$ are independent and identically distributed with $\mathbb{E} X_{i}=0$ and $\mathbb{E} X_{i}^{2}=1$, we have by the central limit theorem (CLT)

$$
\lim _{n \rightarrow+\infty} \sup _{x \in \mathbb{R}}\left|\mathbb{P}\left(S_{n} \leqslant x \sqrt{n}\right)-\phi(x)\right|=0 .
$$

By the theorem of Berry ([1], 1941) and Esseen ([5], 1942), if moreover, $\mathbb{E}\left|X_{i}^{3}\right|<+\infty$, the rate of convergence in the limit is of order $n^{-1 / 2}$. If $\left(X_{i}\right)_{i \in \mathbb{N}}$ is an ergodic martingale difference sequence with $\mathbb{E} X_{i}^{2}=1$, by the theorem of Billingsley ([2], 1968) and Ibragimov (([11], 1963), see also ([10], 1980)) we have the CLT. The rate of convergence can, however, be arbitrarily slow even if $X_{i}$ are bounded and $\alpha$-mixing (cf. [4]). There are several results showing that with certain assumption on the conditional variance $\mathbb{E}\left(X_{i}^{2} \mid \mathcal{F}_{i-1}\right)$, the rate of convergence becomes polynomial (Kato ([12], 1979), Grams ([7], 1972), Nakata ([9], 1976), Bolthausen ([3], 1982), Haeusler ([8], 1988),...).

In 1963, Ibragimov [11] has shown that for $X_{i}$ uniformly bounded, if instead of usual sums $S_{n}$, the stopped sums $S_{v(n)}$ or $S_{v(n)}^{\prime}$ are considered, one gets the rate of convergence of order $n^{-1 / 4}$; the only assumption beside boundedness is that $\sum_{i=0}^{+\infty} \sigma_{i}^{2}$ diverge to infinity a.s.

In the present paper we give a rate of convergence for a larger class of martingale difference sequences, the Ibragimov's case will be a particular one.

\section{Main result}

We consider a sequence $\left(X_{i}\right)_{i \in \mathbb{N}}$ of square integrable martingale differences.

Theorem 1. If the series $\sum_{i=0}^{+\infty} \sigma_{i}^{2}$ diverges a.s. and if there exists a nondecreasing sequence $\left(Y_{i}\right)_{i \in \mathbb{N}}$ adapted to the filtration $\left(\mathcal{F}_{i}, i \in \mathbb{N}\right)$ such that, for all $i \in \mathbb{N}^{*}$

$$
\mathbb{E}\left(Y_{i}^{4}\right)<+\infty, \quad 1 \leqslant Y_{i} \quad \text { and } \quad \mathbb{E}\left(\left|X_{i}\right|^{3} \mid \mathcal{F}_{i-1}\right) \leqslant Y_{i-1} \sigma_{i-1}^{2} \quad \text { a.s. }
$$

then for all $n$ sufficiently large

$$
\begin{aligned}
& \sup _{x \in \mathbb{R}}\left|F_{n}(x)-\phi(x)\right| \leqslant \frac{a_{n}{ }^{1 / 2}}{\pi n^{1 / 4}}\left(11+\frac{3}{4 n^{1 / 4}}+\frac{2}{9 n^{1 / 2}}+\frac{1}{8 n^{3 / 4}}\right), \\
& \sup _{x \in \mathbb{R}}\left|H_{n}(x)-\phi(x)\right| \leqslant \frac{a_{n}{ }^{1 / 2}}{\pi n^{1 / 4}}\left(11+\frac{9}{4 n^{1 / 4}}+\frac{2}{9 n^{1 / 2}}+\frac{1}{8 n^{3 / 4}}\right),
\end{aligned}
$$


where $a_{n}=\left(\mathbb{E} Y_{v(n)}^{4}\right)^{1 / 2}$.

If we put $Y_{i}=M$ a.s. where $M>0$ is a constant, one obtains the following corollaries:

Corollary 1. If the series $\sum_{i=0}^{+\infty} \sigma_{i}^{2}$ diverges a.s. and there exists $M>0$ such that, for all $i \in \mathbb{N}^{*}, \mathbb{E}\left(\left|X_{i}\right|^{3} \mid \mathcal{F}_{i-1}\right) \leqslant$ $M \mathbb{E}\left(X_{i}^{2} \mid \mathcal{F}_{i-1}\right)$ a.s. then there is a constant $0<c_{M}<+\infty$

$$
\begin{aligned}
& \sup _{x \in \mathbb{R}}\left|F_{n}(x)-\phi(x)\right| \leqslant \frac{c_{M}}{n^{1 / 4}}, \\
& \sup _{x \in \mathbb{R}}\left|H_{n}(x)-\phi(x)\right| \leqslant \frac{c_{M}}{n^{1 / 4}} .
\end{aligned}
$$

Corollary 2. If there exists $0<\alpha \leqslant M<+\infty$ satisfying $\sigma_{i-1}^{2} \geqslant \alpha$ and $\mathbb{E}\left(\left|X_{i}\right|^{3} \mid \mathcal{F}_{i-1}\right) \leqslant M$ a.s. for all $i \in \mathbb{N}^{*}$, then there is a constant $0<c_{(\alpha, M)}<+\infty$ such that (4) and (5) hold.

Moreover, if we suppose that $\left(X_{i}\right)_{i \in \mathbb{N}}$ is uniformly bounded, we obtain the result of Ibragimov [11].

Corollary 3. If the series $\sum_{i=0}^{+\infty} \sigma_{i}^{2}$ diverges a.s. and $\left|X_{i}\right| \leqslant M<+\infty$ a.s. for all $i \geqslant 0$, then (4) and (5) hold.

Example. Let $A=\left(A_{k}\right)_{k \in \mathbb{N}}$ be a sequence of real valued random variables such that $\sup _{k \in \mathbb{N}} \mathbb{E}\left(A_{k}^{4}\right)^{1 / 4}=\beta<$ $\infty$ and consider an arbitrary sequence of variables $\zeta=\left(\zeta_{k}\right)_{k \in \mathbb{N}^{*}}$ with zero means, unit variances, bounded third moments and which are also independent of $A$. We define $X=\left(A_{k-1} \zeta_{k}\right)_{k \in \mathbb{N}^{*}}$ and $\mathcal{F}_{k}$ the $\sigma$-algebra generated by $A_{0}, A_{1}, \ldots, A_{k}$.

Clearly $\left(X_{k}, \mathcal{F}_{k}, k \in \mathbb{N}^{*}\right)$ is a martingale difference sequence, and for all $k \in \mathbb{N}^{*}$,

$$
\begin{aligned}
& \mathbb{E}\left(A_{k-1}^{2} \zeta_{k}^{2} \mid \mathcal{F}_{k-1}\right)=A_{k-1}^{2} \quad \text { a.s., } \\
& \mathbb{E}\left(\left|A_{k-1} \zeta_{k}\right|^{3} \mid \mathcal{F}_{k-1}\right) \leqslant\left|A_{k-1}\right| \sup _{i \in \mathbb{N}^{*}} \mathbb{E}\left(\left|\zeta_{i}\right|^{3}\right) A_{k-1}^{2} \quad \text { a.s. }
\end{aligned}
$$

If $\left(\left|A_{k}\right|\right)_{k \in \mathbb{N}}$ is nondecreasing, then using Theorem 1, one obtains

$$
\sup _{x \in \mathbb{R}}\left|F_{n}(x)-\phi(x)\right| \leqslant c \beta \frac{\sup _{k \in \mathbb{N}^{*}} \mathbb{E}\left(\left|\zeta_{k}\right|^{3}\right)^{1 / 4}}{n^{1 / 4}},
$$

where $c$ is a positive constant.

\section{Proof of theorem}

According to Esseen's theorem (see, e.g., ([6], 1954) p. 210 and ([13], 1955) p. 285), for all $y>0$,

$$
\sup _{x \in \mathbb{R}}\left|F_{n}(x)-\phi(x)\right| \leqslant \frac{1}{\pi} \int_{-y}^{y}\left|\mathbb{E}\left\{\exp \left(\frac{i t S_{\nu(n)}}{\sqrt{n}}\right)\right\}-\exp \left(-\frac{t^{2}}{2}\right)\right| \frac{\mathrm{d} t}{|t|}+\frac{24}{\pi \sqrt{2 \pi} y} .
$$

Below we shall prove the following inequalities

$$
\left|\mathbb{E}\left\{\exp \left(\frac{i t S_{\nu(n)}}{\sqrt{n}}+\frac{t^{2}}{2 n} \sum_{p=0}^{v(n)-1} \sigma_{p}^{2}\right)\right\}-1\right| \leqslant a_{n} \mathrm{e}^{\frac{t^{2}}{2}}\left(\frac{|t|}{3 \sqrt{n}}+\frac{t^{2}}{4 n}+\frac{a_{n}|t|^{3}}{3 n^{3 / 2}}+\frac{a_{n} t^{4}}{4 n^{2}}\right),
$$




$$
\begin{aligned}
& \left|\mathbb{E}\left\{\exp \left(\frac{i t S_{v(n)}}{\sqrt{n}}+\frac{t^{2}}{2 n} \sum_{p=0}^{v(n)-1} \sigma_{p}^{2}\right)\right\}-\mathbb{E}\left\{\exp \left(\frac{i t S_{v(n)}}{\sqrt{n}}+\frac{t^{2}}{2}\right)\right\}\right| \leqslant \frac{a_{n} t^{2}}{2 n} \exp \left(\frac{t^{2}}{2}\right), \\
& \left|\mathbb{E}\left\{\exp \left(\frac{i t S_{v(n)}}{\sqrt{n}}\right)\right\}-\mathbb{E}\left\{\exp \left(\frac{i t S_{v(n)}^{\prime}}{\sqrt{n}}\right)\right\}\right| \leqslant \frac{3 a_{n} t^{2}}{2 n}
\end{aligned}
$$

where $a_{n}=\left(\mathbb{E} Y_{v(n)}^{4}\right)^{1 / 2}$.

\subsection{Proof of the inequality (7)}

We have

$$
\begin{aligned}
& \mathbb{E}\left\{\exp \left(\frac{i t S_{\nu(n)}}{\sqrt{n}}+\frac{t^{2}}{2 n} \sum_{p=0}^{\nu(n)-1} \sigma_{p}^{2}\right)\right\}-1 \\
& =\sum_{k=1}^{+\infty} \mathbb{E}\left\{\left(\exp \left(\frac{i t S_{k}}{\sqrt{n}}+\frac{t^{2}}{2 n} \sum_{p=0}^{k-1} \sigma_{p}^{2}\right)-1\right) I_{\nu(n)=k}\right\} \\
& =\sum_{k=1}^{+\infty} \sum_{j=1}^{k} \mathbb{E}\left\{\exp \left(\frac{i t S_{j-1}}{\sqrt{n}}+\frac{t^{2}}{2 n} \sum_{p=0}^{j-1} \sigma_{p}^{2}\right)\left(\mathrm{e}^{\frac{i t x_{j}}{\sqrt{n}}}-\mathrm{e}^{-\frac{t^{2} \sigma_{j-1}^{2}}{2 n}}\right) I_{\nu(n)=k}\right\} .
\end{aligned}
$$

For real $x$, put

$$
\mathrm{e}^{i x}=1+i x+\frac{(i x)^{2}}{2}+u(x), \quad \mathrm{e}^{-x}=1-x+\beta(x) \frac{x^{2}}{2} .
$$

It is easily seen that, for all $x \in \mathbb{R}$

$$
|u(x)| \leqslant \frac{|x|^{3}}{6}, \quad|u(x)| \leqslant \frac{x^{2}}{2}, \quad \text { and } \quad|\beta(|x|)| \leqslant 1 .
$$

Observing that the random variable $W_{j-1}^{n}=\exp \left(\frac{i t S_{j-1}}{\sqrt{n}}+\frac{t^{2}}{2 n} \sum_{p=0}^{j-1} \sigma_{p}^{2}\right)$ is measurable with respect to the $\sigma$-algebra $\mathcal{F}_{j-1}$ and using the identities $(*)$, we obtain

$$
\begin{aligned}
& \mathbb{E}\left\{\exp \left(\frac{i t S_{\nu(n)}}{\sqrt{n}}+\frac{t^{2}}{2 n} \sum_{p=0}^{v(n)-1} \sigma_{p}^{2}\right)\right\}-1 \\
& =\sum_{k=1}^{+\infty} \sum_{j=1}^{k} \mathbb{E}\left\{W_{j-1}^{n} \mathbb{E}\left\{\left(\frac{i t X_{j}}{\sqrt{n}}-\frac{t^{2} X_{j}^{2}}{2 n}+u\left(\frac{t X_{j}}{\sqrt{n}}\right)+\frac{t^{2} \sigma_{j-1}^{2}}{2 n}+\beta\left(\frac{t^{2} \sigma_{j-1}^{2}}{2 n}\right) \frac{t^{4} \sigma_{j-1}^{4}}{8 n^{2}}\right) I_{\nu(n)=k} \mid \mathcal{F}_{j-1}\right\}\right\} .
\end{aligned}
$$

Since $\{v(n)=k\}$ is measurable with respect to the $\sigma$-algebra $\mathcal{F}_{k}$, for all $j \geqslant 2$, we have

$$
\sum_{k=1}^{j-1} \mathbb{E}\left\{X_{j} I_{\nu(n)=k} \mid \mathcal{F}_{j-1}\right\}=\sum_{k=1}^{j-1} \mathbb{E}\left\{\left(X_{j}^{2}-\sigma_{j-1}^{2}\right) I_{\nu(n)=k} \mid \mathcal{F}_{j-1}\right\}=0 .
$$

On the other hand, for all $j \geqslant 1$ we have

$$
\sum_{k=1}^{+\infty} \mathbb{E}\left\{X_{j} I_{\nu(n)=k} \mid \mathcal{F}_{j-1}\right\}=\sum_{k=1}^{+\infty} \mathbb{E}\left\{\left(X_{j}^{2}-\sigma_{j-1}^{2}\right) I_{\nu(n)=k} \mid \mathcal{F}_{j-1}\right\}=0
$$


It follows that, for all $j \geqslant 1$

$$
\sum_{k \geqslant j} \mathbb{E}\left\{X_{j} I_{\nu(n)=k} \mid \mathcal{F}_{j-1}\right\}=\sum_{k \geqslant j} \mathbb{E}\left\{\left(X_{j}^{2}-\sigma_{j-1}^{2}\right) I_{\nu(n)=k} \mid \mathcal{F}_{j-1}\right\}=0 .
$$

So, from (10) we derive

$$
\begin{aligned}
& \left|\mathbb{E}\left\{\exp \left(\frac{i t S_{\nu(n)}}{\sqrt{n}}+\frac{t^{2}}{2 n} \sum_{p=0}^{v(n)-1} \sigma_{p}^{2}\right)\right\}-1\right| \\
& \quad=\left|\sum_{k=1}^{+\infty} \sum_{j=1}^{k} \mathbb{E}\left\{W_{j-1}^{n} \mathbb{E}\left\{\left(u\left(\frac{t X_{j}}{\sqrt{n}}\right)+\beta\left(\frac{t^{2} \sigma_{j-1}^{2}}{2 n}\right) \frac{t^{4} \sigma_{j-1}^{4}}{8 n^{2}}\right) I_{\nu(n)=k} \mid \mathcal{F}_{j-1}\right\}\right\}\right| \\
& \quad \leqslant \sum_{k=1}^{+\infty} \sum_{j=1}^{k} \mathbb{E}\left\{\exp \left(\frac{t^{2}}{2 n} \sum_{p=0}^{j-1} \sigma_{p}^{2}\right) \mathbb{E}\left\{\left(\frac{|t|^{3}\left|X_{j}\right|^{3}}{6 n^{3 / 2}}+\frac{t^{4} \sigma_{j-1}^{4}}{8 n^{2}}\right) I_{\nu(n)=k} \mid \mathcal{F}_{j-1}\right\}\right\} .
\end{aligned}
$$

For any $j \geqslant 2$ and any real function $\psi$ such that $\mathbb{E}\left(\psi\left(X_{k}\right)\right)<\infty$ for any positive $k$, we have

$$
\begin{array}{rl}
\sum_{k=1}^{j-1} & \mathbb{E}\left\{\exp \left(\frac{t^{2}}{2 n} \sum_{p=0}^{j-1} \sigma_{p}^{2}\right) \mathbb{E}\left\{\psi\left(X_{j}\right) I_{\nu(n)=k} \mid \mathcal{F}_{j-1}\right\}\right\} \\
= & \sum_{k=1}^{j-1} \mathbb{E}\left\{\exp \left(\frac{t^{2}}{2 n} \sum_{p=0}^{j-1} \sigma_{p}^{2}\right) \mathbb{E}\left\{\psi\left(X_{j}\right) \mid \mathcal{F}_{j-1}\right\} I_{\nu(n)=k}\right\} .
\end{array}
$$

On the other hand, for all $j \geqslant 1$, we have

$$
\begin{aligned}
& \sum_{k=1}^{+\infty} \mathbb{E}\left\{\exp \left(\frac{t^{2}}{2 n} \sum_{p=0}^{j-1} \sigma_{p}^{2}\right) \mathbb{E}\left\{\psi\left(X_{j}\right) I_{\nu(n)=k} \mid \mathcal{F}_{j-1}\right\}\right\} \\
& =\mathbb{E}\left\{\exp \left(\frac{t^{2}}{2 n} \sum_{p=0}^{j-1} \sigma_{p}^{2}\right) \psi\left(X_{j}\right)\right\} \\
& =\sum_{k=1}^{+\infty} \mathbb{E}\left\{\exp \left(\frac{t^{2}}{2 n} \sum_{p=0}^{j-1} \sigma_{p}^{2}\right) \mathbb{E}\left\{\psi\left(X_{j}\right) \mid \mathcal{F}_{j-1}\right\} I_{\nu(n)=k}\right\} .
\end{aligned}
$$

It follows from (12) and (13) that

$$
\begin{aligned}
\sum_{j=1}^{+\infty} & \sum_{k \geqslant j} \mathbb{E}\left\{\exp \left(\frac{t^{2}}{2 n} \sum_{p=0}^{j-1} \sigma_{p}^{2}\right) \mathbb{E}\left\{\left\{\psi\left(X_{j}\right) I_{\nu(n)=k} \mid \mathcal{F}_{j-1}\right\}\right\}\right. \\
= & \sum_{j=1}^{+\infty} \sum_{k \geqslant j} \mathbb{E}\left\{\exp \left(\frac{t^{2}}{2 n} \sum_{p=0}^{j-1} \sigma_{p}^{2}\right) \mathbb{E}\left\{\psi\left(X_{j}\right) \mid \mathcal{F}_{j-1}\right\} I_{\nu(n)=k}\right\} .
\end{aligned}
$$

Applying (11) and (14) for $\psi(x)=|x|^{3}$ we deduce that

$$
\left|\mathbb{E}\left\{\exp \left(\frac{i t S_{\nu(n)}}{\sqrt{n}}+\frac{t^{2}}{2 n} \sum_{p=0}^{v(n)-1} \sigma_{p}^{2}\right)\right\}-1\right|
$$




$$
\begin{aligned}
& \leqslant \sum_{k=1}^{+\infty} \sum_{j=1}^{k} \mathbb{E}\left\{\exp \left(\frac{t^{2}}{2 n} \sum_{p=0}^{j-1} \sigma_{p}^{2}\right)\left(\mathbb{E}\left\{\frac{|t|^{3}\left|X_{j}\right|^{3}}{6 n^{3 / 2}} \mid \mathcal{F}_{j-1}\right\} I_{\nu(n)=k}+\mathbb{E}\left\{\frac{t^{4} \sigma_{j-1}^{4}}{8 n^{2}} I_{\nu(n)=k} \mid \mathcal{F}_{j-1}\right\}\right)\right\} \\
& \leqslant \sum_{k=1}^{+\infty} \sum_{j=1}^{k} \mathbb{E}\left\{\exp \left(\frac{t^{2}}{2 n} \sum_{p=0}^{j-1} \sigma_{p}^{2}\right)\left(\frac{|t|^{3} Y_{j-1} \sigma_{j-1}^{2}}{6 n^{3 / 2}} I_{\nu(n)=k}+\frac{t^{4} \sigma_{j-1}^{4}}{8 n^{2}} I_{\nu(n)=k}\right)\right\} .
\end{aligned}
$$

By the Hölder inequality, for all $j \in \mathbb{N}^{*}$

$$
\sigma_{j-1}^{2}=\mathbb{E}\left(X_{j}^{2} \mid \mathcal{F}_{j-1}\right) \leqslant \mathbb{E}\left(\left|X_{j}\right|^{3} \mid \mathcal{F}_{j-1}\right)^{2 / 3} \leqslant Y_{j-1}^{2 / 3} \sigma_{j-1}^{4 / 3} \quad \text { a.s. }
$$

whence

$$
\sigma_{j-1}^{2} \leqslant Y_{j-1}^{2} \quad \text { a.s. }
$$

From (15), (16) and using the fact that $Y_{k} \geqslant Y_{j-1} \geqslant 1$ for all $j \leqslant k$, we deduce that

$$
\begin{aligned}
& \left|\mathbb{E}\left\{\exp \left(\frac{i t S_{v(n)}}{\sqrt{n}}+\frac{t^{2}}{2 n} \sum_{p=0}^{v(n)-1} \sigma_{p}^{2}\right)\right\}-1\right| \\
& \leqslant\left(\frac{|t|^{3}}{6 n^{3 / 2}}+\frac{t^{4}}{8 n^{2}}\right) \sum_{k=1}^{+\infty} \sum_{j=1}^{k} \mathbb{E}\left\{Y_{j-1}^{2} \sigma_{j-1}^{2} \exp \left(\frac{t^{2}}{2 n} \sum_{p=0}^{j-1} \sigma_{p}^{2}\right) I_{\nu(n)=k}\right\} \\
& \leqslant\left(\frac{|t|^{3}}{6 n^{3 / 2}}+\frac{t^{4}}{8 n^{2}}\right) \sum_{k=1}^{+\infty} \mathbb{E}\left\{Y_{k}^{2} \sum_{j=1}^{k} \sigma_{j-1}^{2} \exp \left(\frac{t^{2}}{2 n} \sum_{p=0}^{j-1} \sigma_{p}^{2}\right) I_{\nu(n)=k}\right\} .
\end{aligned}
$$

To bound up the terms appearing in (17), we will use the following elementary lemma.

Lemma 1. Let $k \geqslant 1$, then on the event $\{v(n)=k\}$ we have

$$
\sum_{j=1}^{k} \exp \left(\frac{t^{2}}{2 n} \sum_{p=0}^{j-1} \sigma_{p}^{2}\right) \frac{t^{2}}{2 n} \sigma_{j-1}^{2} \leqslant \exp \left(\frac{t^{2}}{2}\right)\left(1+\frac{Y_{k}^{2} t^{2}}{n}\right) .
$$

Proof. On the event $\{v(n)=k\}$, we have

$$
\begin{aligned}
\exp \left(\frac{t^{2}}{2}\right) & \geqslant \exp \left(\frac{t^{2}}{2 n} \sum_{p=0}^{k-1} \sigma_{p}^{2}\right)-\exp \left(\frac{t^{2}}{2 n} \sigma_{0}^{2}\right) \\
& \geqslant \sum_{j=1}^{k-1} \exp \left(\frac{t^{2}}{2 n} \sum_{p=0}^{j-1} \sigma_{p}^{2}\right)\left(\exp \left(\frac{t^{2} \sigma_{j}^{2}}{2 n}\right)-1\right) .
\end{aligned}
$$

Using the inequality, $\exp (x)-1 \geqslant x$ for all $x \geqslant 0$, one obtains

$$
\exp \left(\frac{t^{2}}{2}\right) \geqslant \sum_{j=1}^{k-1} \exp \left(\frac{t^{2}}{2 n} \sum_{p=0}^{j-1} \sigma_{p}^{2}\right) \frac{t^{2}}{2 n} \sigma_{j}^{2}
$$

Therefore

$$
\sum_{j=1}^{k-1} \exp \left(\frac{t^{2}}{2 n} \sum_{p=0}^{j-1} \sigma_{p}^{2}\right) \frac{t^{2}}{2 n} \sigma_{j-1}^{2}
$$




$$
\begin{aligned}
\leqslant & \sum_{j=1}^{k-1} \exp \left(\frac{t^{2}}{2 n} \sum_{p=0}^{j-1} \sigma_{p}^{2}\right) \frac{t^{2}}{2 n}\left(\sigma_{j-1}^{2}-\sigma_{j}^{2}\right)+\exp \left(\frac{t^{2}}{2}\right) \\
= & \sum_{j=1}^{k-2}\left(\exp \left(\frac{t^{2}}{2 n} \sum_{p=0}^{j} \sigma_{p}^{2}\right)-\exp \left(\frac{t^{2}}{2 n} \sum_{p=0}^{j-1} \sigma_{p}^{2}\right)\right) \frac{t^{2}}{2 n} \sigma_{j}^{2}-\frac{t^{2}}{2 n} \exp \left(\frac{t^{2}}{2 n} \sum_{p=0}^{k-2} \sigma_{p}^{2}\right) \sigma_{k-1}^{2} \\
& +\frac{t^{2}}{2 n} \exp \left(\frac{t^{2}}{2 n} \sigma_{0}^{2}\right) \sigma_{0}^{2}+\exp \left(\frac{t^{2}}{2}\right) \\
\leqslant & \frac{t^{2}}{2 n} Y_{k}^{2} \sum_{j=1}^{k-2}\left(\exp \left(\frac{t^{2}}{2 n} \sum_{p=0}^{j} \sigma_{p}^{2}\right)-\exp \left(\frac{t^{2}}{2 n} \sum_{p=0}^{j-1} \sigma_{p}^{2}\right)\right)+\frac{t^{2}}{2 n} Y_{k}^{2} \exp \left(\frac{t^{2}}{2 n} \sigma_{0}^{2}\right)+\exp \left(\frac{t^{2}}{2}\right) \\
\leqslant & \left(1+\frac{t^{2}}{2 n} Y_{k}^{2}\right) \exp \left(\frac{t^{2}}{2}\right) .
\end{aligned}
$$

We conclude the proof of the lemma by noting that $\sigma_{k-1}^{2} \leqslant Y_{k}^{2}$ and $\sum_{p=0}^{k-1} \sigma_{p}^{2} \leqslant n$ a.s.

Finally, according to Lemma 1 and the (17) we get

$$
\left|\mathbb{E}\left\{\exp \left(\frac{i t S_{v(n)}}{\sqrt{n}}+\frac{t^{2}}{2 n} \sum_{p=0}^{v(n)-1} \sigma_{p}^{2}\right)\right\}-1\right| \leqslant a_{n} \exp \left(\frac{t^{2}}{2}\right)\left(\frac{|t|}{3 \sqrt{n}}+\frac{t^{2}}{4 n}+\frac{a_{n}|t|^{3}}{3 n^{3 / 2}}+\frac{a_{n} t^{4}}{4 n^{2}}\right),
$$

where $a_{n}=\left(\mathbb{E} Y_{v(n)}^{4}\right)^{1 / 2}$.

\subsection{Proof of the inequality (8)}

Using (1) and the inequality $|1-\exp (-x)| \leqslant x$, for all $x \geqslant 0$ we see that

$$
\begin{aligned}
& \left|\mathbb{E}\left\{\exp \left(\frac{i t S_{v(n)}}{\sqrt{n}}+\frac{t^{2}}{2 n} \sum_{p=0}^{v(n)-1} \sigma_{p}^{2}\right)\right\}-\mathbb{E}\left\{\exp \left(\frac{i t S_{v(n)}}{\sqrt{n}}+\frac{t^{2}}{2}\right)\right\}\right| \\
& \quad=\left|\mathbb{E}\left\{\exp \left(\frac{i t S_{v(n)}}{\sqrt{n}}+\frac{t^{2}}{2}\right)\left(\exp \left(-\frac{t^{2}}{2 n} \gamma(n) \sigma_{v(n)}^{2}\right)-1\right)\right\}\right| \\
& \leqslant \mathbb{E}\left\{\left|1-\exp \left(-\frac{t^{2}}{2 n} \gamma(n) \sigma_{v(n)}^{2}\right)\right|\right\} \exp \left(\frac{t^{2}}{2}\right) \\
& \leqslant \mathbb{E}\left\{\frac{t^{2}}{2 n}|\gamma(n)| \sigma_{v(n)}^{2}\right\} \exp \left(\frac{t^{2}}{2}\right) \\
& \leqslant\left(\mathbb{E} Y_{v(n)}^{4}\right)^{1 / 2} \frac{t^{2}}{2 n} \exp \left(\frac{t^{2}}{2}\right) .
\end{aligned}
$$

Therefore (8) holds true.

From (7) and (8) we conclude that

$$
\left|\mathbb{E}\left\{\exp \left(\frac{i t S_{\nu(n)}}{\sqrt{n}}\right)\right\}-\exp \left(-\frac{t^{2}}{2}\right)\right| \leqslant a_{n}\left(\frac{|t|}{3 \sqrt{n}}+\frac{3 t^{2}}{4 n}+\frac{|t|^{3}}{3 n^{3 / 2}} a_{n}+\frac{t^{4}}{4 n^{2}} a_{n}\right) .
$$

Using Esseen's theorem, we derive

$$
\sup _{x \in \mathbb{R}}\left|F_{n}(x)-\phi(x)\right| \leqslant \frac{a_{n}}{\pi} \int_{-y}^{y}\left(\frac{1}{3 \sqrt{n}}+\frac{3|t|}{4 n}+\frac{t^{2}}{3 n^{3 / 2}} a_{n}+\frac{|t|^{3}}{4 n^{2}} a_{n}\right) \mathrm{d} t+\frac{24}{\pi \sqrt{2 \pi} y} .
$$


Hence

$$
\sup _{x \in \mathbb{R}}\left|F_{n}(x)-\phi(x)\right| \leqslant \frac{a_{n}}{\pi}\left(\frac{2 y}{3 \sqrt{n}}+\frac{3 y^{2}}{4 n}+\frac{2 y^{3}}{9 n^{3 / 2}} a_{n}+\frac{y^{4}}{8 n^{2}} a_{n}\right)+\frac{24}{\pi \sqrt{2 \pi} y} .
$$

Choosing $y$ in such a way that $y / \sqrt{n}=1 /\left(y a_{n}\right)$, i.e. $y=\left(n / a_{n}^{2}\right)^{1 / 4}$, we infer that

$$
\sup _{x \in \mathbb{R}}\left|F_{n}(x)-\phi(x)\right| \leqslant \frac{a_{n}^{1 / 2}}{\pi n^{1 / 4}}\left(11+\frac{3}{4 n^{1 / 4}}+\frac{2}{9 n^{1 / 2}}+\frac{1}{8 n^{3 / 4}}\right) .
$$

The proof of the inequality (2) in theorem is complete.

\subsection{Proof of the inequality (9)}

Observing that the random events $\{\gamma(n) \leqslant x\} \cap\{v(n)=k\}$ and consequently the random variables $\sqrt{\gamma(n)} I_{v(n)=k}$ are measurable with respect to $\mathcal{F}_{k}$, we find that

$$
\begin{aligned}
\mid \mathbb{E} & \left\{\exp \left(\frac{i t S_{\nu(n)}}{\sqrt{n}}\right)\right\}-\mathbb{E}\left\{\exp \left(\frac{i t S_{v(n)}^{\prime}}{\sqrt{n}}\right)\right\} \mid \\
& =\left|\sum_{k=0}^{+\infty} \mathbb{E}\left\{\left(\exp \left(\frac{i t S_{k}}{\sqrt{n}}\right)-\exp \left(\frac{i t S_{k}}{\sqrt{n}}+\frac{i t \sqrt{\gamma(n)}}{\sqrt{n}} X_{\nu(n)+1}\right)\right) I_{\nu(n)=k}\right\}\right| \\
& \leqslant \sum_{k=0}^{+\infty}\left|\mathbb{E}\left\{\exp \left(\frac{i t S_{k}}{\sqrt{n}}\right)\left(1-\exp \left(\frac{i t \sqrt{\gamma(n)}}{\sqrt{n}} X_{\nu(n)+1}\right)\right) I_{\nu(n)=k}\right\}\right| \\
& =\sum_{k=0}^{+\infty}\left|\mathbb{E}\left\{\exp \left(\frac{i t S_{k}}{\sqrt{n}}\right)\left(-\frac{i t \sqrt{\gamma(n)}}{\sqrt{n}} X_{k+1}+\frac{t^{2}}{2 n} \gamma(n) X_{k+1}^{2}-u\left(\frac{t \sqrt{\gamma(n)}}{\sqrt{n}} X_{k+1}\right)\right) I_{\nu(n)=k}\right\}\right| \\
& =\sum_{k=0}^{+\infty}\left|\mathbb{E}\left\{\exp \left(\frac{i t S_{k}}{\sqrt{n}}\right) \mathbb{E}\left\{-\frac{i t \sqrt{\gamma(n)}}{\sqrt{n}} X_{k+1}+\frac{t^{2}}{2 n} \gamma(n) X_{k+1}^{2}-u\left(\frac{t \sqrt{\gamma(n)} X_{k+1}}{\sqrt{n}}\right) \mid \mathcal{F}_{k}\right\} I_{\nu(n)=k}\right\}\right| \\
& =\sum_{k=0}^{+\infty}\left|\mathbb{E}\left\{\exp \left(\frac{i t S_{k}}{\sqrt{n}}\right)\left(\frac{t^{2}}{2 n} \gamma(n) X_{k+1}^{2}-u\left(\frac{t}{\sqrt{n}} \sqrt{\gamma(n)} X_{k+1}\right)\right) I_{\nu(n)=k}\right\}\right| \\
& \leqslant \sum_{k=0}^{+\infty} \mathbb{E}\left\{I_{\nu(n)=k} \frac{3 t^{2}}{2 n} \gamma(n) X_{k+1}^{2}\right\} \\
& \leqslant \frac{3 t^{2}}{2 n} \sum_{k=0}^{+\infty} \mathbb{E}\left\{I_{v(n)=k} \mathbb{E}\left\{X_{k+1}^{2} \mid \mathcal{F}_{k}\right\}\right\} \\
& \leqslant \frac{3 t^{2}}{2 n} \mathbb{E}\left(Y_{v(n)}^{4}\right)^{1 / 2} .
\end{aligned}
$$

The proof of the inequality (9) is complete.

\subsection{Proof of the inequality (3)}

According to Esseen's theorem where $y=\left(n / a_{n}^{2}\right)^{1 / 4}$ and the inequality (9), one obtains 


$$
\begin{aligned}
\sup _{x \in \mathbb{R}}\left|H_{n}(x)-\phi(x)\right| & \leqslant \frac{1}{\pi} \int_{-y}^{y}\left|\mathbb{E}\left\{\exp \left(\frac{i t S_{v(n)}^{\prime}}{\sqrt{n}}\right)\right\}-\exp \left(-\frac{t^{2}}{2}\right)\right| \frac{\mathrm{d} t}{|t|}+\frac{24}{\pi \sqrt{2 \pi} y} \\
& \leqslant \frac{a_{n}^{1 / 2}}{\pi n^{1 / 4}}\left(11+\frac{3}{4 n^{1 / 4}}+\frac{2}{9 n^{1 / 2}}+\frac{1}{8 n^{3 / 4}}\right)+\frac{1}{\pi} \int_{-y}^{y} \frac{3|t|}{2 n} E\left(Y_{v(n)}^{4}\right)^{1 / 2} \mathrm{~d} t \\
& \leqslant \frac{a_{n}{ }^{1 / 2}}{\pi n^{1 / 4}}\left(11+\frac{3}{4 n^{1 / 4}}+\frac{2}{9 n^{1 / 2}}+\frac{1}{8 n^{3 / 4}}\right)+\frac{3}{2 \pi \sqrt{n}} \\
& \leqslant \frac{a_{n}{ }^{1 / 2}}{\pi n^{1 / 4}}\left(11+\frac{9}{4 n^{1 / 4}}+\frac{2}{9 n^{1 / 2}}+\frac{1}{8 n^{3 / 4}}\right) .
\end{aligned}
$$

The proof of theorem is complete.

Proofs of Corollaries 1, 2 and 3 are easy so, it is left to the reader.

\section{Acknowledgement}

The author thanks the referee for careful reading of the manuscript and for valuable suggestions which improved the presentation of this paper.

\section{References}

[1] A.C. Berry, The accuracy of the Gaussian approximation to the sum of independent variates, Trans. Amer. Math. Soc. 49 (1941) $122-136$.

[2] P. Billingsley, Convergence of Probability Measures, Wiley, 1968.

[3] E. Bolthausen, Exact convergence rates in some martingale central limit theorems, Ann. Probab. 10 (3) (1982) $672-688$.

[4] M. El Machkouri, D. Volný, On the central and local limit theorems for martingale difference sequences, Stochastics and Dynamics, submitted for publication.

[5] C.G. Esseen, On the Liapounoff limit of error in the theory of probability, Ark. Math. Astr. och Fysik A 28 (1942) 1-19.

[6] B.V. Gnedenko, A.N. Kolomogorov, Limit Distributions for Sums of Independent Random Variables, Addison-Wesley, Reading, MA, 1954, translated by K.L. Chung.

[7] W.F. Grams, Rate of convergence in the central limit theorem for dependent variables, Dissertation, Florida State Univ., 1972.

[8] E. Haeusler, On the rate of convergence in the central limit theorem for martingales with discrete and continuous time, Ann. Probab. 16 (1) (1988) 275-299.

[9] T. Nakata, On the rate of convergence in mean central limit theorem for martingale differences, Rep. Statist. Appl. Res. Un. Japan. Sci. Engrs. 23 (1976) 10-15.

[10] P. Hall, C.C. Heyde, Martingale Limit Theory and Its Application, Academic Press, New York, 1980.

[11] I.A. Ibragimov, A central limit theorem for a class of dependent random variables, Theory Probab. Appl. 8 (1963) 83-89.

[12] Y. Kato, Rates of convergence in central limit theorem for martingale differences, Bull. Math. Statist. 18 (1979) 1-8.

[13] M. Loève, Probability Theory, D. Van Nostrand, Princeton, NJ, 1955. 